\title{
Ethnic variations in glucose, maternal weight and fetal overgrowth in a multi-ethnic inner city antenatal cohort
}

\author{
R Agha-Jaffar ${ }^{1}$, W J Lo ${ }^{1}$, H Shaikh ${ }^{2}$, S Misra ${ }^{1}$, J Terry ${ }^{3}$, A McCarthy ${ }^{3}$, C Yu ${ }^{3}$, B Jones ${ }^{3}$, \\ D Gable ${ }^{2}$, A Dornhorst ${ }^{2}$, Godsland ${ }^{1}$, KGMM Alberti ${ }^{1}$ N Oliver ${ }^{1}$ \& S Robinson ${ }^{2}$ \\ 1. Division of Diabetes, Endocrinology and Metabolism, Imperial College London, UK \\ 2. Department of Metabolic Medicine and 3. Department of Obstetrics and Gynaecology, Imperial College NHS Trust, London, UK
}

\section{Background and Aims}

- The Hyperglycaemia and Adverse Outcomes in Pregnancy Study (HAPO) group demonstrated a continuum between glucose and proportion of infants born large for gestational age (LGA: $\geq 90^{\text {th }}$ birth weight centile).

-We aimed to determine if this varied according to ethnicity.

\section{Methods}

- Pregnant women who underwent a $24-28$ week $75 \mathrm{~g}$ oral glucose tolerance test in an inner-city healthcare centre were retrospectively analysed $(n=4562)$.

- Baseline maternal demographics, glycaemia and fetal birthweight/ birthweight centile were compared across five ethnic groups (Figure 1).

- Variations in the proportion of LGA infants by category of glycaemia and body mass index (BMI) were examined.

Figure 1: Categorisation of ethnicity
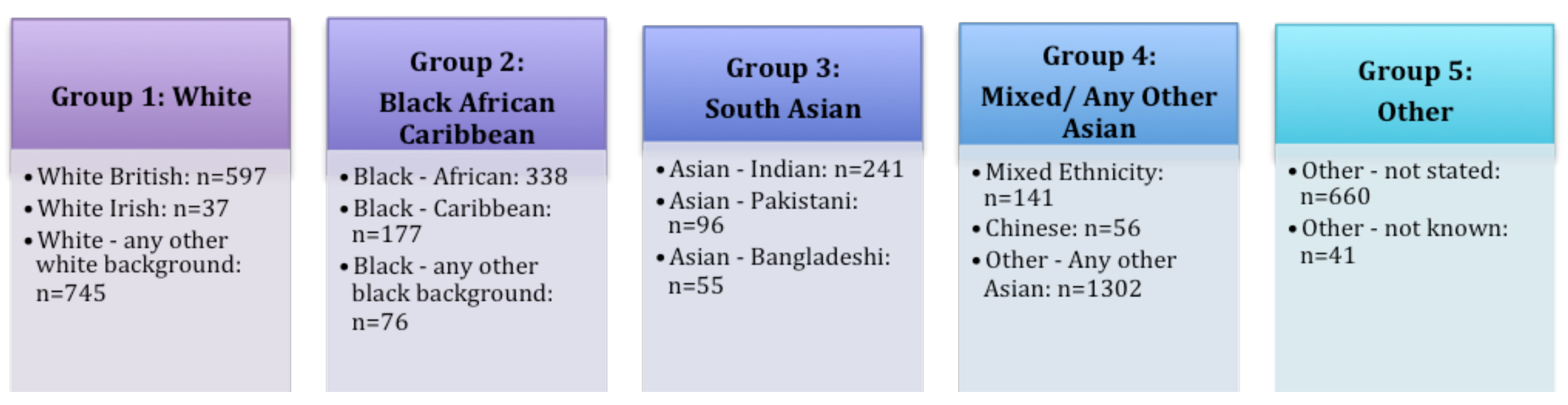

\section{Results}

- Significant differences were observed in maternal age, early pregnancy $\mathrm{BMI}$, fasting plasma glucose (FPG), 120-minute value glucose, fetal birthweight and birthweight centile across the groups.

Table 1: Numbers diagnosed with gestational diabetes

\begin{tabular}{|c|c|c|c|c|c|c|}
\hline & $\begin{array}{l}\text { Group 1: } \\
\text { White } \\
\text { Caucasian }\end{array}$ & $\begin{array}{l}\text { Group 2: } \\
\text { Black } \\
\text { African- } \\
\text { Caribbean }\end{array}$ & $\begin{array}{l}\text { Group 3: } \\
\text { South Asian }\end{array}$ & $\begin{array}{l}\text { Group 4: } \\
\text { Mixed/ Any } \\
\text { Other }\end{array}$ & $\begin{array}{l}\text { Group 5: } \\
\text { Otherl Not } \\
\text { Known }\end{array}$ & $P$ value \\
\hline$N$ & 1379 & 591 & 392 & 1499 & 701 & \\
\hline $\begin{array}{l}\text { Mean }(\mathrm{SD}) \text { age } \\
\quad \text { (years) }\end{array}$ & $\begin{array}{c}34.1 \\
( \pm 5.3)\end{array}$ & $\begin{array}{c}30.8 \\
( \pm 6.0)\end{array}$ & $\begin{array}{c}31.9 \\
( \pm 4.2)\end{array}$ & $\begin{array}{c}32.0 \\
( \pm 5.5)\end{array}$ & $\begin{array}{c}32.2 \\
( \pm 5.6)\end{array}$ & $<0.001$ \\
\hline $\begin{array}{l}\text { Mean (SD) BMI } \\
\left(\mathrm{kg} / \mathrm{m}^{2}\right)\end{array}$ & $\begin{array}{c}25.5 \\
( \pm 6.1)\end{array}$ & $\begin{array}{c}28.1 \\
( \pm 6.0)\end{array}$ & $\begin{array}{l}25.4 \\
( \pm 4.9)\end{array}$ & $\begin{array}{c}25.4 \\
( \pm 5.8)\end{array}$ & $\begin{array}{c}25.5 \\
( \pm 5.3)\end{array}$ & $<0.001$ \\
\hline $\begin{array}{l}\text { Primigravida \% } \\
\text { (n) }\end{array}$ & $\begin{array}{l}53.4 \\
(736)\end{array}$ & $\begin{array}{l}40.4 \\
(239)\end{array}$ & $\begin{array}{l}49.8 \\
(195)\end{array}$ & $\begin{array}{l}54.5 \\
(817)\end{array}$ & $\begin{array}{l}60.6 \\
(425)\end{array}$ & $<0.001$ \\
\hline $\begin{array}{l}\text { Proportion with } \\
\text { GDM \% (n) }\end{array}$ & $\begin{array}{l}5.1 \\
(70)\end{array}$ & $\begin{array}{l}8.1 \\
(48)\end{array}$ & $\begin{array}{l}13.0 \\
(51)\end{array}$ & $\begin{array}{c}9.3 \\
(140)\end{array}$ & $\begin{array}{l}8.0 \\
(56)\end{array}$ & $<0.001$ \\
\hline $\begin{array}{c}\text { Mean (SD) FPG } \\
(\mathrm{mmol} / \mathrm{L})\end{array}$ & $\begin{array}{c}4.33 \\
( \pm 0.46)\end{array}$ & $\begin{array}{c}4.34 \\
( \pm 0.66)\end{array}$ & $\begin{array}{c}4.47 \\
( \pm 0.69)\end{array}$ & $\begin{array}{c}4.40 \\
( \pm 0.68)\end{array}$ & $\begin{array}{c}4.31 \\
( \pm 0.70)\end{array}$ & $<0.001$ \\
\hline $\begin{array}{l}\text { Mean (SD) } 120 \\
\text { min glucose } \\
(\mathrm{mmol} / \mathrm{L})\end{array}$ & $\begin{array}{c}5.42 \\
( \pm 1.36)\end{array}$ & $\begin{array}{c}5.54 \\
( \pm 1.52)\end{array}$ & $\begin{array}{c}6.09 \\
( \pm 1.74)\end{array}$ & $\begin{array}{c}5.69 \\
( \pm 1.58)\end{array}$ & $\begin{array}{c}5.60 \\
( \pm 1.55)\end{array}$ & $<0.001$ \\
\hline $\begin{array}{c}\text { Mean (SD) } \\
\text { Birthweight (g) }\end{array}$ & $\begin{array}{c}3391 \\
( \pm 562)\end{array}$ & $\begin{array}{c}3266 \\
( \pm 551)\end{array}$ & $\begin{array}{c}3074 \\
( \pm 552)\end{array}$ & $\begin{array}{c}3269 \\
( \pm 546)\end{array}$ & $\begin{array}{c}3294 \\
( \pm 524)\end{array}$ & $<0.001$ \\
\hline $\begin{array}{c}\text { Median (IQR) } \\
\text { fetal birth weight } \\
\text { centile }\end{array}$ & $\begin{array}{c}47 \\
(25-75)\end{array}$ & $\begin{array}{c}35 \\
(16-62)\end{array}$ & $\begin{array}{c}25 \\
(10-49)\end{array}$ & $\begin{array}{c}37 \\
(16-64)\end{array}$ & $\begin{array}{c}36 \\
(16-62)\end{array}$ & $<0.001$ \\
\hline
\end{tabular}

\section{Results}

- The proportion of infants born LGA increased with each increment in FPG category in four of the ethnic groups but not in the Other/ Unknown group (Figure 2).

- No clear pattern emerged between 120-minute glucose values and LGA incidence.

- An overall trend towards an increasing proportion of LGA infants with each $5.0 \mathrm{~kg} / \mathrm{m}^{2}$ increment in BMI was observed (Figure 3)

Figure 2: Proportion of infants born large for gestational age $\left(\geq 90^{\text {th }}\right.$ centile) in each ethnic group by category of fasting plasma glucose

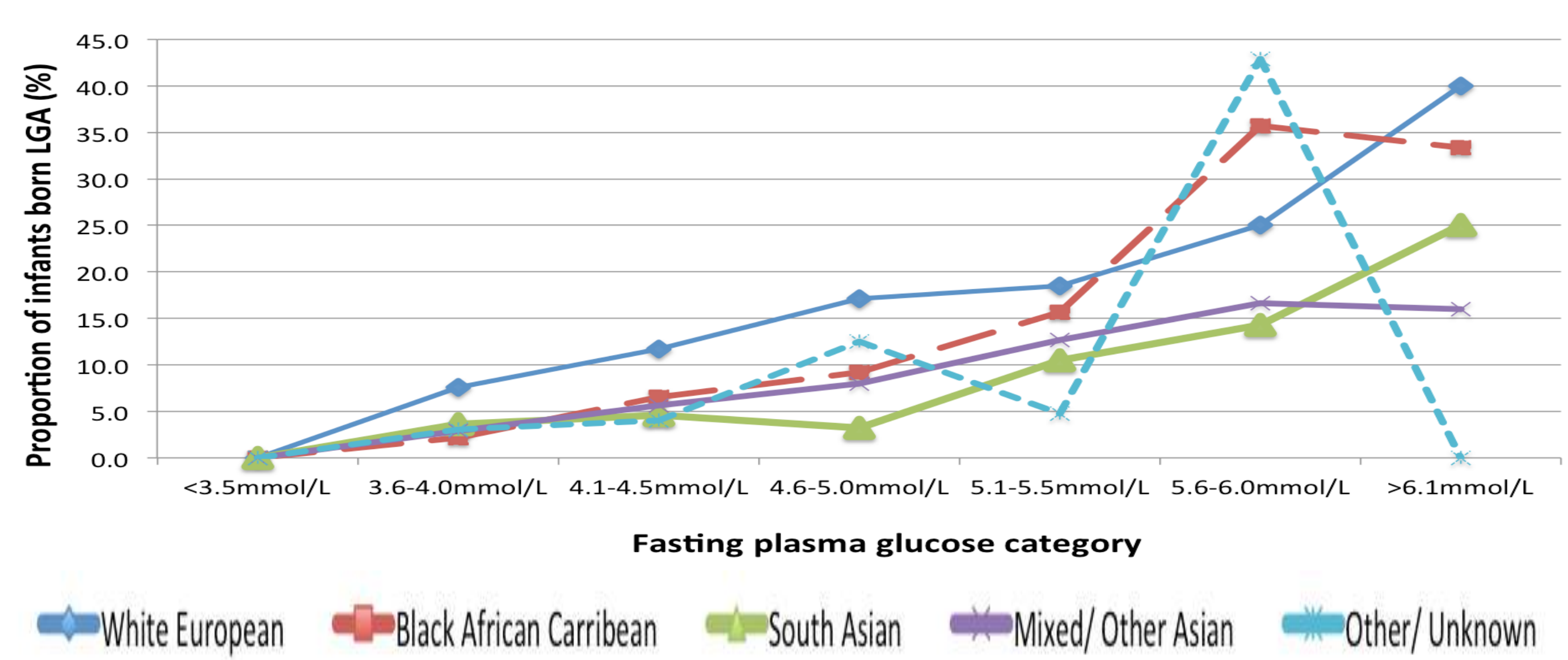

Figure 3: Proportion of infants born large for gestational age $\left(\geq 90^{\text {th }}\right.$ centile) in each ethnic group by early pregnancy body mass index category

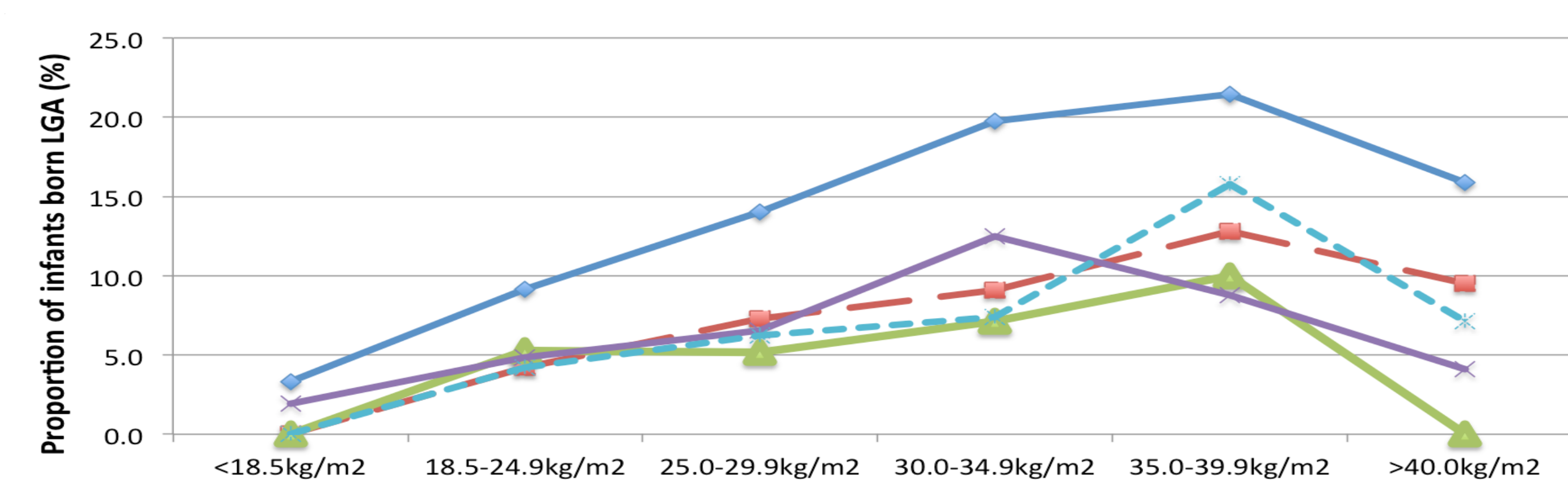

Early pregnancy body mass index category

\section{Results}

- Regression analyses were used to determine if an interaction between glucose, early pregnancy BMI and fetal birthweight existed that was dependent on ethnicity.

- Following adjustment for gestational age and GDM diagnosis, a $1 \mathrm{mmol} / \mathrm{L}$ increase in FPG increased birthweight by $157 \mathrm{~g}(\mathrm{p}<0.001)$ : this interaction was ethnic group dependent (effect size significantly smaller in women of Mixed ethnicity/ other Asian or Other/ Unknown group).

- Interactions between 120-minute glucose value and fetal birthweight were also dependent on ethnicity with black African-Caribbean women exhibiting the largest effect size (27.6g increase in birthweight for every $1 \mathrm{mmol} / \mathrm{L}$ increase in glucose).

\section{Conclusions and discussion}

- Data suggest impact of glucose and BMI on fetal overgrowth varies according to ethnicity.

- An ethnic group dependent effect on the interaction between glucose and fetal birthweight is suggested.

- Ethnicity did not impact on the interaction with early pregnancy BMI and fetal birthweight.

- Ethnicity specific glucose thresholds for the diagnosis of GDM may be warranted 